\title{
Antiferromagnetic cavity optomagnonics
}

\author{
T. S. Parvini* and V. A. S. V. Bittencourt ${ }^{\dagger}$ \\ Max Planck Institute for the Science of Light, Staudtstrasse 2, PLZ 91058 Erlangen, Germany \\ Silvia Viola Kusminskiy ${ }^{\ddagger}$ \\ Max Planck Institute for the Science of Light, Staudtstrasse 2, PLZ 91058 Erlangen, Germany \\ and Institute for Theoretical Physics, University Erlangen-Nürnberg, Staudtstrasse 7, 91058 Erlangen, Germany
}

(Received 29 August 2019; accepted 8 April 2020; published 5 May 2020)

\begin{abstract}
Currently there is a growing interest in studying the coherent interaction between magnetic systems and electromagnetic radiation in a cavity, prompted partly by possible applications in hybrid quantum systems. We propose a multimode cavity optomagnonic system based on antiferromagnetic insulators, where optical photons couple coherently to the two homogeneous magnon modes of the antiferromagnet. These have frequencies typically in the $\mathrm{THz}$ range, a regime so far mostly unexplored in the realm of coherent interactions, and which makes antiferromagnets attractive for quantum transduction from $\mathrm{THz}$ to optical frequencies. We derive the theoretical model for the coupled system, and show that it presents unique characteristics. In particular, if the antiferromagnet presents hard-axis magnetic anisotropy, the optomagnonic coupling can be tuned by a magnetic field applied along the easy axis. This allows us to bring a selected magnon mode into and out of a dark mode, providing an alternative for a quantum memory protocol. The dynamical features of the driven system present unusual behavior due to optically induced magnon-magnon interactions, including regions of magnon heating for a red-detuned driving laser. The multimode character of the system is evident in a substructure of the optomagnonically induced transparency window.
\end{abstract}

DOI: 10.1103/PhysRevResearch.2.022027

Introduction. The interaction between light and magnetism at the quantum level holds promise for future information technologies. In seminal recent experiments, the coherent coupling of magnons (the spin-wave quanta) to optical photons has been demonstrated in solid state optomagnonic cavities [1-3]. These are dielectric magnetic structures capable of simultaneously confining light and magnons, providing an enhancement of the magnon-photon coupling and enabling the study of cavity effects in a new platform. Magnons in these structures exhibit good coherence properties and tunable frequencies, and can couple strongly to microwave (MW) cavity fields [4-10]. Quantum memories [11-18] or transducers allowing for coherent information transfer between MW and optics are envisioned applications $[19,20]$.

Exciting developments have also emerged very recently in the $\mathrm{THz}$ regime, a frequency range which has been historically challenging due to the absence of efficient sources and detectors (the "THz gap") [21]. Strong light-matter coupling

\footnotetext{
*tahereh.parvini@mpl.mpg.de

†victor.bittencourt@mpl.mpg.de

‡silvia.viola-kusminskiy@mpl.mpg.de
}

Published by the American Physical Society under the terms of the Creative Commons Attribution 4.0 International license. Further distribution of this work must maintain attribution to the author(s) and the published article's title, journal citation, and DOI. Open access publication funded by the Max Planck Society. in the $\mathrm{THz}$ regime has been achieved employing cavities [22-24], including strong coupling to magnons in antiferromagnets (AFMs) [25,26], opening the door for quantum applications in the $\mathrm{THz}$ domain. Antiferromagnetic materials support magnons that can be described as excitations of a spin antialigned ground state (the Néel state) [27]. Their frequencies are typically in the $\mathrm{THz}$ range, making AFMs ideal candidates to incorporate in $\mathrm{THz}$ platforms [28-31]. The physics of AFMs in combination with electromagnetic cavities is just starting to be explored. Besides the mentioned experiments in the $\mathrm{THz}$ regime $[25,26]$, strong coupling between MW photons and AFM magnons has been reported [32], while magnon dark modes [33] and coupling to ferromagnets [34] via a MW cavity have been proposed theoretically. In turn, methods involving light to probe and control AFMs are being developed $[35,36]$. These developments are a great incentive to study the coupling of AFM magnons to optical cavities, which could lead to quantum transducers from the $\mathrm{THz}$ to the optical regime.

In this Rapid Communication, we propose a cavity optomagnonic system based on an antiferromagnetic insulator (see Fig. 1). Optomagnonic cavities have been investigated so far exclusively within the scope of ferromagnetic (FM) magnons with $\mathrm{GHz}$ frequencies, both experimentally [1-3,37-39] [although the material of choice, yttrium iron garnet (YIG), is a ferrimagnet, one sublattice has a much larger spin and is dominant] and theoretically [40-47]. We show that for an AFM, different phenomenology emerges. We derive the Hamiltonian governing the system and show that, in the presence of 


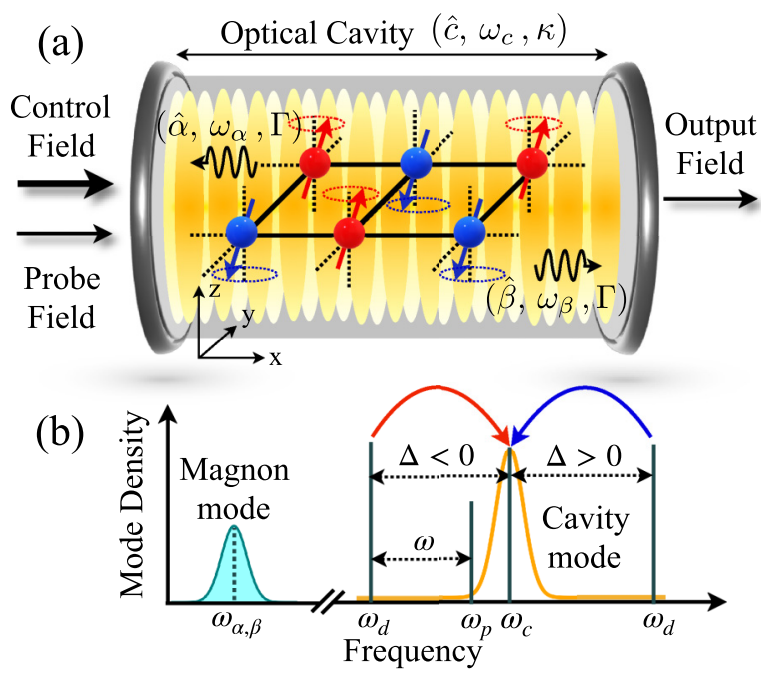

FIG. 1. (a) Schematics of the antiferromagnetic optomagnonic cavity. The homogeneous magnon modes $\hat{\alpha}$ and $\hat{\beta}$ with frequencies $\omega_{\alpha, \beta}$ and decay rates $\Gamma_{\alpha, \beta}$ couple to a cavity mode $\hat{c}$ with frequency $\omega_{c}$ and decay rate $\kappa$. (b) Pump-probe scheme: $\Omega_{m}, \omega_{c}, \omega_{d}, \omega_{p}$, magnon, optical cavity resonance, drive, and probe frequencies, respectively. The detuning of the drive is $\Delta=\omega_{d}-\omega_{c}$, and $\omega=$ $\omega_{p}-\omega_{d}$.

hard-axis anisotropy, the optomagnonic coupling to both supported homogeneous magnon modes can be tuned by an external magnetic field. In particular, we show that the magnon modes can be selectively decoupled from the cavity, rendering them dark. This is unique to AFM cavity optomagnonics. Based on this tunability, we sketch a quantum memory protocol. We further characterize the dynamical response of the system and show that the cavity-mediated interaction between the AFM magnon modes leads to unusual optically induced magnon cooling and heating.

Model. We consider an AFM insulator with two magnetic sublattices $A$ and $B$ of opposite spin. The AFM hosts two homogeneous magnon modes $\alpha$ and $\beta$ (see Fig. 1), and we assume it acts also as an optical cavity by total internal reflection, analogous to dielectric optomechanical [48] or optomagnonic [1-3] cavities. AFMs with a high index of refraction and low absorption in the optical range, such as $\mathrm{NiO}$ $(n \approx 2.4)$ [49] would serve the purpose, or heterostructures containing $\mathrm{MnF}_{2}(n \approx 1.4)$ [50] or $\mathrm{FeF}_{2}(n \approx 1.5)$ [51]. The Hamiltonian of the coupled system is

$$
\hat{\mathcal{H}}=\hat{\mathcal{H}}_{\mathrm{ph}}+\hat{\mathcal{H}}_{\mathrm{AFM}}+\hat{\mathcal{H}}_{\mathrm{OM}},
$$

with $\hat{\mathcal{H}}_{\mathrm{ph}}$ and $\hat{\mathcal{H}}_{\mathrm{AFM}}$ the free photonic and AFM Hamiltonians, respectively. $\hat{\mathcal{H}}_{\mathrm{OM}}$ contains the coupling between the AFM magnons and the cavity photons, and is our main result in this section.

The quantized optical field in the cavity is $\hat{\mathbf{E}}(\mathbf{r}, t)=$ $1 / 2 \sum_{\xi}\left[\mathbf{E}_{\xi}(\mathbf{r}) \hat{c}_{\xi}(t)+\mathbf{E}_{\xi}^{*}(\mathbf{r}) \hat{c}_{\xi}^{\dagger}(t)\right]$, with $\hat{c}_{\xi}^{(\dagger)}$ the annihilation (creation) operator of mode $\xi$ with resonance frequency $\omega_{\xi}$, hence $\hat{\mathcal{H}}_{\mathrm{ph}}=\hbar \sum_{\xi} \omega_{\xi} \hat{c}_{\xi}^{\dagger} \hat{c}_{\xi} \cdot \hat{\mathcal{H}}_{\mathrm{AFM}}$ consists of (i) the exchange interaction between nearest-neighbor spins $J \sum_{\langle i \neq j\rangle} \hat{\mathbf{S}}_{i} \cdot \hat{\mathbf{S}}_{j}$ $(J>0)$, (ii) the Zeeman interaction between spins and an external dc magnetic field $\boldsymbol{B}_{0}$ along $\boldsymbol{e}_{z},|\gamma| B_{0} \sum_{i} \hat{\mathbf{S}}_{i}^{z}(\gamma$ gyromagnetic ratio), and (iii) easy-axis $-\frac{K_{\|}}{2} \sum_{i}\left(S_{i}^{z}\right)^{2}\left(K_{\|}>\right.$ $0)$ and hard-axis $\frac{K_{\perp}}{2} \sum_{i}\left(S_{i}^{x}\right)^{2}\left(K_{\perp} \geqslant 0\right)$ anisotropy in the $\boldsymbol{e}_{z}$ and $\boldsymbol{e}_{x}$ directions, respectively. For small magnetization fluctuations around the Néel ordered state, the HolsteinPrimakoff (HP) transformations [52,53] can be used to express $\hat{\mathcal{H}}_{\mathrm{AFM}}$ in terms of bosonic operators $\hat{a}_{\boldsymbol{k}}$ and $\hat{b}_{\boldsymbol{k}}$ associated with the sublattices $A$ and $B$. To first order, the HP transformations are given in terms of spin ladder operators as $\hat{S}_{A(B)}^{+}=\sqrt{2 S / N} \sum_{\boldsymbol{k}} e^{-i \boldsymbol{k} \cdot \boldsymbol{x}_{i}} \hat{a}_{\boldsymbol{k}}\left(\hat{b}_{\boldsymbol{k}}^{\dagger}\right)$, where $N$ is the total number of sites per sublattice and $S$ the spin on each site. $\hat{\mathcal{H}}_{\mathrm{AFM}}$ is diagonalized via a four-dimensional (4D) Bogoliubov transformation to the bosonic operators $\hat{\alpha}_{\boldsymbol{k}}=u_{\alpha, a} \hat{a}_{\boldsymbol{k}}+v_{\alpha, b} \hat{b}_{-\boldsymbol{k}}^{\dagger}+v_{\alpha, a} \hat{a}_{-\boldsymbol{k}}^{\dagger}+u_{\alpha, b} \hat{b}_{\boldsymbol{k}}$ and $\hat{\beta}_{\boldsymbol{k}}=u_{\beta, a} \hat{a}_{\boldsymbol{k}}+$ $v_{\beta, b} \hat{b}_{-\boldsymbol{k}}^{\dagger}+v_{\beta, a} \hat{a}_{-\boldsymbol{k}}^{\dagger}+u_{\beta, b} \hat{b}_{\boldsymbol{k}}$ [see Supplemental Material (SM) [54]]: $\hat{\mathcal{H}}_{\mathrm{AFM}}=\hbar \sum_{\boldsymbol{k}}\left[\omega_{\alpha \boldsymbol{k}} \hat{\alpha}_{\boldsymbol{k}}^{\dagger} \hat{\alpha}_{\boldsymbol{k}}+\omega_{\beta \boldsymbol{k}} \hat{\beta}_{\boldsymbol{k}}^{\dagger} \hat{\beta}_{\boldsymbol{k}}\right]$, with $\omega_{\alpha, \beta \boldsymbol{k}}$ the respective eigenfrequencies. We restrict our analysis to the two homogeneous $(\boldsymbol{k}=0)$ AFM magnon modes, hence from hereon we drop the index $\boldsymbol{k}$. The corresponding magnon frequencies $\omega_{\alpha, \beta}$ are functions of the characteristic frequencies $\omega_{E}=\hbar J S N, \omega_{\|, \perp}=\hbar S N K_{\|, \perp}$, and $\omega_{H}=|\gamma| B_{0}: \omega_{\alpha(\beta)}^{2}=\omega_{H}^{2}+\omega_{E} \omega_{\perp}+2 \omega_{E} \omega_{\|}+\omega_{\perp} \omega_{\|}+\omega_{\|}^{2} \pm$ $\sqrt{\omega_{E}^{2} \omega_{\perp}^{2}+4 \omega_{E} \omega_{H}^{2}\left(\omega_{\perp}+2 \omega_{\|}\right)+\omega_{H}^{2}\left(\omega_{\perp}+2 \omega_{\|}\right)^{2}}[34,55,56]$. Note that $\omega_{\alpha} \geqslant \omega_{\beta}$ and hence $\alpha(\beta)$ labels the upper (lower) mode. Whereas $\omega_{\alpha}$ increases with the magnetic field, $\omega_{\beta}$ decreases and goes to zero at the onset of the spin-flop phase at $\omega_{H}=\omega_{\mathrm{SF}} \approx \sqrt{2 \omega_{E} \omega_{\|}}$[57].

The interaction between light and magnetization is described by the magneto-optical coupling $\mathcal{H}_{\mathrm{OM}}=$ $\sum_{\mu, v} \int d \boldsymbol{r} E_{\mu}^{*}(\boldsymbol{r}) \varepsilon_{\mu \nu}\left(S_{\boldsymbol{r}}\right) E_{v}(\boldsymbol{r}) / 4$, where $\varepsilon_{\mu \nu} \quad(\mu, v=x, y, z)$ is the spin-dependent part of the permittivity tensor. In this Rapid Communication we consider simple cubic and rutile-structure AFMs, and other more complex structures will be considered elsewhere. For these materials, within linear response in the deviations from the magnetic equilibrium, $\mathcal{H}_{\text {OM }}$ reduces to $[54,58,59]$

$$
\begin{aligned}
\mathcal{H}_{\mathrm{OM}}= & \frac{K_{+} V}{4 N} \sum_{i \in A, B}\left(P_{i}^{+} S_{-}^{i}-P_{i}^{-} S_{+}^{i}\right) \\
& +\frac{K_{-} V}{4 N}\left[\sum_{i \in A}\left(P_{i}^{+} S_{+}^{i}-P_{i}^{-} S_{-}^{i}\right)\right. \\
& \left.-\sum_{j \in B}\left(P_{j}^{+} S_{+}^{j}-P_{j}^{-} S_{-}^{j}\right)\right],
\end{aligned}
$$

where we have discretized the interaction and $P_{i}^{ \pm}=$ $E_{z}^{*}\left(\boldsymbol{r}_{i}\right) E_{ \pm}\left(\boldsymbol{r}_{i}\right)-E_{\mp}^{*}\left(\boldsymbol{r}_{i}\right) E_{z}\left(\boldsymbol{r}_{i}\right)$, with $E_{ \pm}=E_{x} \pm i E_{y}$ and $S_{ \pm}=$ $S_{x} \pm i S_{y}$. The linear magneto-optic coefficients $K_{ \pm}$correspond to processes in which the two sublattices scatter the light in phase $(+)$ or out of phase $(-)$. For our purposes, one-magnon processes coming from quadratic terms in the spin (e.g., $\propto \hat{S}^{z} \hat{S}^{ \pm}$) can be absorbed in the definition of $K_{ \pm}$. This model applies, e.g., to the uniaxial $\mathrm{AFMs}_{\mathrm{MnF}}$ or $\mathrm{FeF}_{2}$ [60,61], and for the simple cubic AFM NiO for which $K_{-}=0$.

We obtain the optomagnonic coupling Hamiltonian $\hat{\mathcal{H}}_{\mathrm{OM}}$ by quantizing Eq. (2) assuming that the electric field varies smoothly, such that $P_{i}^{ \pm} \approx P_{j}^{ \pm}$for nearest neighbors. We focus 
on the interaction between the homogeneous AFM magnon modes $\hat{\alpha}$ and $\hat{\beta}$ with a single optical mode $\hat{c}$ with frequency $\omega_{c}$. For an optical mode with circular polarization in the $y z$ plane, from Eq. (2) we obtain [54]

$$
\hat{\mathcal{H}}_{\mathrm{OM}}=-\hbar G \hat{c}^{\dagger} \hat{c}\left(g_{\alpha} \hat{\alpha}^{\dagger}+g_{\beta} \hat{\beta}^{\dagger}+\text { H.c. }\right),
$$

with ( $\varepsilon$ is the AFM dielectric constant)

$$
G=\frac{\omega_{c} K_{+}}{8 \varepsilon} \sqrt{\frac{2 S}{N}} .
$$

The AFM optomagnonic coupling depends on the Bogoliubov coefficients through

$$
g_{\alpha(\beta)}=\left(u_{\alpha(\beta)}^{+}+v_{\alpha(\beta)}^{+}\right)+K\left(u_{\alpha(\beta)}^{-}+v_{\alpha(\beta)}^{-}\right),
$$

where $K=K_{-} / K_{+}$quantifies the intrinsic magneto-optical asymmetry between the sublattices, and we have defined $u_{\alpha(\beta)}^{ \pm}=u_{a, \alpha(\beta)} \pm u_{b, \alpha(\beta)}$ and $v_{\alpha(\beta)}^{ \pm}=v_{a, \alpha(\beta)} \pm v_{b, \alpha(\beta)}$. Hence, the two AFM magnon modes $\hat{\alpha}$ and $\hat{\beta}$ couple, in general, with different strengths to the cavity mode.

Optomagnonic coupling. The constant $G$ describes the coupling to the magnetization's fluctuation sector and is consistent with the one derived in Ref. [44] for the optomagnonic coupling in a ferromagnetically ordered system. Assuming equivalent sublattices with Faraday rotation per unit length $\theta_{\mathrm{F}}$, then $K_{+}=c \sqrt{\varepsilon} \theta_{\mathrm{F}} /\left(\omega_{c} S\right)$ (with $c$ the speed of light) and thus $G=(1 / \sqrt{2 N S})\left(c \theta_{\mathrm{F}} / 4 \sqrt{\varepsilon}\right)$. The $1 / \sqrt{N}$ dependence indicates that the density of excitations is relevant for the coupling, favoring small magnetic volumes. Due to the lack of data on absolute values for $K_{+}$(or $\theta_{\mathrm{F}}$ ) for simple AFMs, we take as an estimate for $G$ the value for $(1 \mu \mathrm{m})^{3}$ YIG (diffraction limit volume), $G_{\mathrm{YIG}}=0.1 \mathrm{MHz}$ [44]. Some measurements indicate that the Faraday rotation coefficient in AFMs can be quite large, e.g., similar values as for YIG have been reported for $\mathrm{BiFeO}_{3}$ [62]. Note that $G$ given in Eq. (4) assumes perfect mode matching. Imperfect mode overlap can be accounted for by a mode-volume ratio factor [44] and it is responsible for a suppression of the coupling in current experiments with YIG $[19,63]$. The second term in Eq. (5) gives a contribution proportional to $K G$ and describes the coupling to fluctuations of the Néel vector. Typical values of $K$ are $K \approx 0.01$ (e.g., for $\mathrm{MnF}_{2}$ or $\mathrm{FeF}_{2}$ [60]).

The reduced couplings $g_{\alpha, \beta}$ can be found analytically, but the general solution is lengthy. Simple expressions can be given in certain cases. Since the exchange energy is usually the largest energy scale in the AFM, the condition $\omega_{\perp, \|} \ll \omega_{E}$ holds. For an easy-axis AFM $\left(\omega_{\perp}=0\right)$, we obtain [54]

$$
g_{\alpha, \beta}^{\omega_{\perp}=0} \approx\left(\frac{\omega_{\|}}{2 \omega_{E}}\right)^{1 / 4} \pm K\left(\frac{2 \omega_{E}}{\omega_{\|}}\right)^{1 / 4} .
$$

Equation (6) is independent of the magnetic field $B_{0}$, a consequence of the axial symmetry of the system in this case [54]. In the absence of magneto-optical asymmetry $(K=0)$ both modes couple equally to the light field, while for finite $K$, $g_{\alpha}^{\omega_{\perp}=0}\left(g_{\beta}^{\omega_{\perp}=0}\right)$ increases (decreases) linearly. If the condition $K=\sqrt{\omega_{\|} / 2 \omega_{E}}$ is met, $g_{\beta}^{\omega_{\perp}=0}=0$ and $\beta$ is a dark mode, completely decoupled from the cavity. Whereas this requires fine tuning, it could be achievable in cold atom realizations where the relevant parameters can be tuned [64-68]. The situation nevertheless changes in the presence of hard-axis anisotropy,

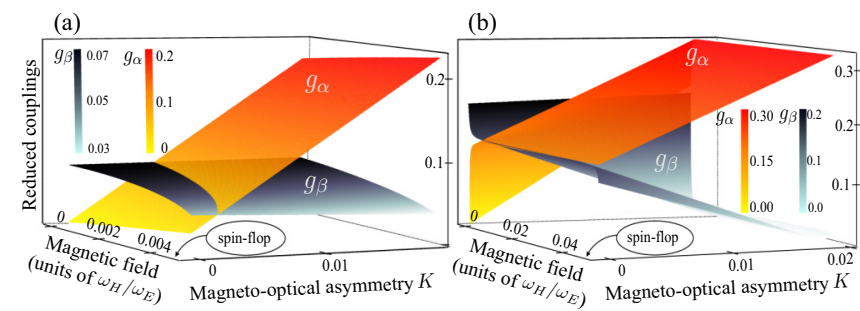

FIG. 2. Reduced optomagnonic coupling coefficients $g_{\alpha}$ and $g_{\beta}$, as a function of external bias magnetic field (as $\omega_{H} / \omega_{E}$ ) and of the magneto-optical asymmetry $K$. (a) Hard-axis dominated regime $\left(\omega_{\perp}>\omega_{\|}\right), \omega_{\|} / \omega_{E}=1.3 \times 10^{-5}, \omega_{\perp} / \omega_{E}=7.6 \times 10^{-4}(\mathrm{NiO}[71])$. (b) Easy-axis dominated regime $\left(\omega_{\|}>\omega_{\perp}\right), \omega_{\perp} / \omega_{E}=1.3 \times 10^{-5}$, $\omega_{\|} / \omega_{E}=7.6 \times 10^{-4}$.

where the coupling to the modes can be tuned externally by the magnetic field as we show below. From Eq. (6) we obtain $g_{\alpha, \beta}^{\mathrm{MnF}_{2}} \approx 0.5,0.4\left(\omega_{E}=9.3 \mathrm{THz}, \omega_{\|}=0.15 \mathrm{THz}, K=0.007\right.$ $[60,69])$ and $g_{\alpha, \beta}^{\mathrm{FeF}_{2}} \approx 0.6,0.7\left(\omega_{E}=9.5 \mathrm{THz}, \omega_{\|}=3.5 \mathrm{THz}\right.$, $K=0.01[59,70])$.

In the absence of a magnetic field, $\hat{\mathcal{H}}_{\mathrm{AFM}}$ is invariant under $\hat{a}_{k} \longleftrightarrow \hat{b}_{-k}$. For finite hard-axis anisotropy $\left(\omega_{\perp} \neq 0\right)$, imposing this symmetry we obtain [54]

$$
\begin{aligned}
& g_{\alpha}^{\omega_{H}=0, \omega_{\perp} \neq 0}=2 K\left(u_{\alpha, a}-v_{\alpha, a}\right), \\
& g_{\beta}^{\omega_{H}=0, \omega_{\perp} \neq 0}=2\left(u_{\beta, b}-v_{\beta, b}\right),
\end{aligned}
$$

and hence for $K=0 \hat{\alpha}$ is a dark mode $\left(g_{\alpha}=0\right)$ while $\hat{\beta}$ is independent of $K$. The case $\omega_{\perp}=B_{0}=0$ is, however, pathological, since $\hat{\alpha}$ and $\hat{\beta}$ are degenerate. Then Eq. (6) holds, with $g_{\alpha}=g_{\beta} \neq 0$ (the Bogoliubov coefficients present a discontinuity at $\omega_{\perp}=0$ ).

Figure 2 shows $\left|g_{\alpha}\right|$ and $\left|g_{\beta}\right|$ as a function of $B_{0}$ and $K$ for representative finite anisotropy values $\omega_{\perp}$ and $\omega_{\|}$. In Fig. 2(a) we took these as for $\mathrm{NiO}$ [71], and in Fig. 2(b) we exchanged them such that $\omega_{\|}>\omega_{\perp}$. In both cases the coupling strengths $g_{\alpha, \beta}$ can be tuned by $B_{0}$, although with some qualitative differences. For $K=0$ the $\alpha$ mode can be tuned from dark to bright by increasing $B_{0}$, with a slow linear increase for $\omega_{\|}<\omega_{\perp}$ and rapidly but saturating for $\omega_{\|}>\omega_{\perp}$. For both cases there is a threshold $K_{\mathrm{th}}$ such that for $K>K_{\mathrm{th}}$, there exists a finite $B_{0}$ for which the $\beta$ mode is rendered dark $\left(g_{\beta}=0\right)$. In the regime considered for Fig. $2, g_{\alpha, \beta}<1$ for all fields, since the maximum $B_{0}$ is limited by the spin-flop transition. This suppresses the corresponding optomagnonic coupling $\left(G g_{\alpha, \beta}\right) . g_{\alpha}$ increases nevertheless rapidly with $K$, so materials with a larger magneto-optical asymmetry would be favorable for larger coupling values. Our calculations indicate that $K \gtrsim 0.1$ would be sufficient for $g_{\alpha}>1$ [54].

A figure of merit for determining the strength of the coupling is the cooperativity [48]. Taking $G=0.1 \mathrm{MHz}$ as noted above, and typical values for the magnon $(\Gamma \approx 1 \mathrm{GHz}$ $[72,73])$ and optical cavity decay rates $(\kappa \approx 100 \mathrm{MHz}[1])$, for $g_{\alpha, \beta}=1$ we obtain a single-photon cooperativity $\mathcal{C}_{\alpha, \beta}^{0}=$ $4 G^{2} g_{\alpha, \beta}^{2} / \Gamma \kappa \approx 4 \times 10^{-6}$. For an estimated maximum photon density of $10^{5} / \mu \mathrm{m}^{3}$ allowed in the cavity [44], the cooperativity $\mathcal{C}_{\alpha, \beta}=n_{c} \mathcal{C}_{\alpha, \beta}^{0}$ (with $n_{c}=\left\langle\hat{c}^{\dagger} \hat{c}\right\rangle$ the steady state number of photons circulating in the cavity) could be therefore tuned into 

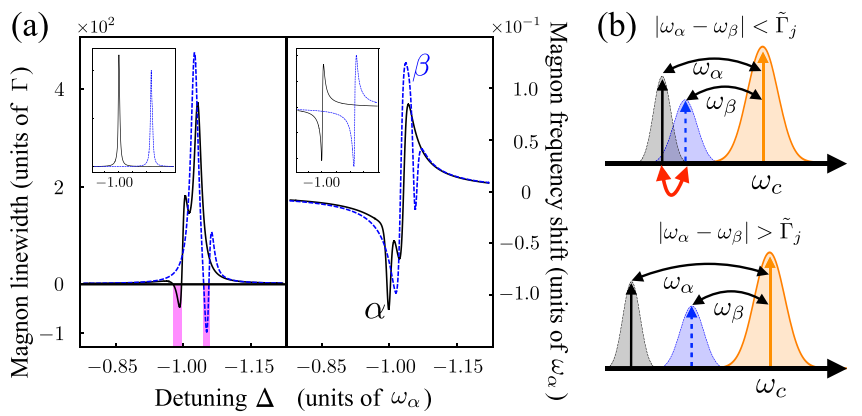

FIG. 3. (a) Effective linewidth (left) and frequency shift (right) of the magnon modes vs detuning $\Delta / \omega_{\alpha}$ for near-degenerate modes and (inset) well-separated modes. The highlighted area indicates an unusual amplification region in the red-detuned regime. (b) Frequency scheme for near-degenerate and well-separated frequencies. The red arrow indicates indirect magnon-magnon interactions, more relevant in the near-degenerate case. Parameters for $\mathrm{MnF}_{2}$ and $\Gamma / \omega_{E}=1.6 \times$ $10^{-4}, \kappa / \omega_{E}=3.7 \times 10^{-3}, \omega_{H} / \omega_{E}=5.4 \times 10^{-3}$ for the main figures and $\omega_{H} / \omega_{E}=3.2 \times 10^{-2}$ for the inset.

the strong-coupling regime $\left(\mathcal{C}_{\alpha, \beta}>1\right)$ by reaching $g_{\alpha, \beta}>1$. Improved cavity and magnon decay rates would boost this value further. In this regime, magnons and photons hybridize and a coherent exchange of information is possible.

Dynamical response. We now consider a cavity driven by a strong control laser with amplitude $s_{d}$ and frequency $\omega_{d}$, and a weak probe laser with amplitude $s_{p}$ and frequency $\omega_{p}$ (see Fig. 1). Correspondingly, we add a driving term $\hat{\mathcal{H}}_{\mathrm{D}}=$ $i \hbar \sqrt{\eta \kappa}\left(\hat{c}_{\xi}^{\dagger} s_{\text {in }}+\right.$ H.c. $)$ to the Hamiltonian in Eq. (1), where $s_{\text {in }}=s_{d} e^{-i \omega_{l} t}+s_{p} e^{-i \omega_{p} t}$. The total loss rate of the optical cavity is $\kappa=\kappa_{\mathrm{ex}}+\kappa_{0}$, where $\kappa_{\mathrm{ex}}$ and $\kappa_{0}$ correspond to the loss rates due to external coupling and intrinsic dissipation, respectively. The coupling efficiency $\eta=\kappa_{\mathrm{ex}} / \kappa_{0}$ is adjustable in experiments $[74,75]$.

The cavity leads to the modification of both the magnon resonance frequency and the magnon damping. Both effects are quantified through the magnon self-energy, which also includes a cavity-mediated coupling between the two magnon modes. This term becomes relevant in the strong-coupling regime $\left(g_{j} \sqrt{n_{c}}>\Gamma_{j}, \kappa\right.$, with $j=\alpha, \beta$ and $\Gamma_{j}$ the magnon linewidth of mode $j$ ) for near-degenerate magnon modes $\left|\omega_{\alpha}-\omega_{\beta}\right|<\Gamma_{j}$ (see SM [54]). Together with hybridization effects [76-78] and counter-rotating terms that cannot be neglected in this regime, the optically induced magnon-magnon interaction is responsible for unusual behavior, for example, amplification in the red-detuned regime (see Fig. 3). The AFM cavity provides a unique platform to probe such regimes for materials that exhibit degenerate modes at zero magnetic field (such as $\mathrm{MnF}_{2}$ ), since $\left|\omega_{\alpha}-\omega_{\beta}\right|$ can be tuned via an external magnetic field.

We now turn our attention to the transmission and reflection properties of the AFM optomagnonic cavity. Following the standard procedure (see SM [54]) we obtain the cavity mode spectra $\delta c[\omega]$ in the frame rotating at the control light frequency,

$$
\delta c[\omega]=\frac{[1+F(\omega)] \sqrt{\eta \kappa} \delta s_{\text {in }}[\omega]}{-i(\tilde{\Delta}+\omega)+\frac{\kappa}{2}-2 i \tilde{\Delta} F(\omega)},
$$

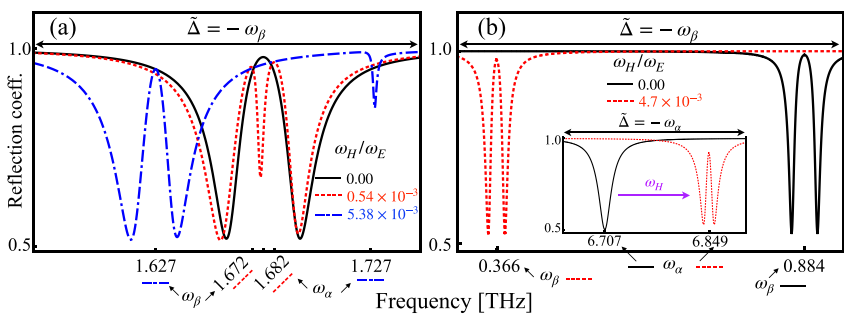

FIG. 4. Reflection spectra for a red-detuned control laser as a function of the probe-pump detuning $\omega$ for a material with (a) degenerate magnon modes at zero magnetic field and (b) nondegenerate modes. Parameters: $\eta=0.25, \kappa=3.5 \times 10^{-2} \mathrm{THz}, \Gamma=1.5 \times$ $10^{-3} \mathrm{THz}$, (a) parameters for $\mathrm{MnF}_{2}$ and $G \sqrt{n_{c}} / \omega_{E}=4.0 \times 10^{-3}$, and (b) parameters for $\mathrm{NiO}$ and $G \sqrt{n_{c}} / \omega_{E}=1.8 \times 10^{-3}$.

where $\omega=\omega_{p}-\omega_{d}$ is the pump-probe detuning, $\tilde{\Delta}=$ $\Delta+2 G\left(g_{\alpha} \operatorname{Re}[\langle\hat{\alpha}\rangle]+g_{\beta} \operatorname{Re}[\langle\hat{\beta}\rangle]\right)$ is the renormalized detuning due to the magnon-induced cavity frequency shift with $\langle\hat{j}\rangle=i G g_{j} n_{c} /\left(i \omega_{j}+\Gamma_{j} / 2\right)$ (for $\left.j=\alpha, \beta\right)$, and $F(\omega)=$ $\Sigma(\omega) /[i(\tilde{\Delta}-\omega)+\kappa / 2]$ with the photon self-energy term $\Sigma(\omega)=\Sigma_{\alpha}(\omega)+\Sigma_{\beta}(\omega)$ given in terms of

$$
\Sigma_{j}(\omega)=\left[\frac{G^{2}\left|g_{j}\right|^{2} n_{c}}{-i\left(\omega_{j}+\omega\right)+\frac{\Gamma_{j}}{2}}-\frac{G^{2}\left|g_{j}\right|^{2} n_{c}}{i\left(\omega_{j}-\omega\right)+\frac{\Gamma_{j}}{2}}\right] .
$$

The transmission and reflection spectra are obtained from Eq. (7) by using the input-output boundary conditions $\delta c_{\text {out }}(\omega)=\delta c_{\text {in }}(\omega)+\left(\kappa_{\text {ex }} / 2\right)^{1 / 2} \delta c(\omega)$. In Fig. 4 we plot the reflection spectra for the fast-cavity regime $\left(\Gamma<g_{\alpha} G \sqrt{n_{c}}<\right.$ $\kappa$ and for simplicity we assume $\left.\Gamma_{\alpha}=\Gamma_{\beta} \equiv \Gamma\right)$. Due to destructive interference between the up-converted control field and the probe field, an optomagnonically induced transparency (OMIT) window opens in the transmission spectrum around the corresponding magnon resonance. In the neardegenerate regime, depicted in Fig. 4(a) for representative parameters of $\mathrm{MnF}_{2}$ (easy-axis AFM), the OMIT window has an additional structure due to the closeness of the $\alpha$ and $\beta$ modes' sidebands. Increasing $B_{0}$ increases $\left|\omega_{\beta}-\omega_{\alpha}\right|$ [but has no effect on the optomagnonic coupling - see Eq. (6)] and the usual OMIT behavior is recovered. In Fig. 4(b) we show results for representative parameters of $\mathrm{NiO}$ (finite hard-axis anisotropy), for which the magnon frequencies are well separated even at zero magnetic field. In this case the OMIT behavior can be tuned by $B_{0}$ through the optomagnonic coupling (see Fig. 2).

Finally, the dark-to-bright tunability of the magnon modes can be used for a quantum memory protocol. Driving the system with a strong control red-detuned laser, the cavitymagnon coupling can be controlled by $B_{0}$ such that the (linearized) Hamiltonian is $\sim g(t)\left(\delta \hat{c}^{\dagger} \hat{\alpha}+\delta \hat{c} \hat{\alpha}^{\dagger}\right)$ [48]. An arbitrary initial cavity state can then be stored in the magnon mode by bringing $g(t)$ from its initial value $g_{0}$ to $g(T)=0$ such that $\int_{0}^{T} d \operatorname{tg}(t)=\pi g_{0}$ (analogous to a $\pi$-pulse protocol [79]). This swaps the state of the cavity with the magnon mode, which is then rendered dark for $t>T$. The state can be transferred with high fidelity for strong coupling and $T \gg$ $1 / \kappa$, and stored up to the magnon lifetime. Alternatively, the 
OMIT could be employed, in a similar fashion to memories implemented in cold atoms [80-84]. The AFM permits us to tune the OMIT window via $B_{0}$, allowing a broad bandwidth storage.

Conclusions. We proposed a solid state optomagnonic cavity system in which optical photons are coupled to longwavelength AFM magnons and derived its governing Hamiltonian and dynamical features. We showed that the AFM system presents unique characteristics, such as tunability of the coupling with a magnetic field, and unusual dynamical effects due to cavity-induced interactions between the two homogeneous magnon modes. We estimated the values for the coupling and showed that, although challenging, the strongcoupling regime could be reached in micron-sized singledomain AFM cavities [85,86]. AFM optical cavities could therefore provide another platform to study light-matter interactions, and possibly an alternative tool to probe AFMs due to the enhanced light-magnon coupling. The tunability with a magnetic field, in particular, for tuning a magnon mode from dark to bright, shows promise for quantum protocols for quantum information storage and retrieval. The coherent coupling of $\mathrm{THz}$ magnons to optical photons could allow the implementation of a quantum transducer [26]. Here, we focused on one-magnon processes; two-magnon processes will be treated elsewhere.

Acknowledgments. The authors acknowledge the financial support from the Max Planck Gesellschaft through an independent Max Planck Research group. We thank A. Aiello, T. Nova, L. Petruzziello, L. Smaldone, and C. Tzschaschel for enlightening discussions.
[1] X. Zhang, N. Zhu, C.-L. Zou, and H. X. Tang, Phys. Rev. Lett. 117, 123605 (2016).

[2] A. Osada, R. Hisatomi, A. Noguchi, Y. Tabuchi, R. Yamazaki, K. Usami, M. Sadgrove, R. Yalla, M. Nomura, and Y. Nakamura, Phys. Rev. Lett. 116, 223601 (2016).

[3] J. A. Haigh, A. Nunnenkamp, A. J. Ramsay, and A. J. Ferguson, Phys. Rev. Lett. 117, 133602 (2016).

[4] Ö. O. Soykal and M. E. Flatté, Phys. Rev. Lett. 104, 077202 (2010).

[5] H. Huebl, C. W. Zollitsch, J. Lotze, F. Hocke, M. Greifenstein, A. Marx, R. Gross, and S. T. B. Goennenwein, Phys. Rev. Lett. 111, 127003 (2013)

[6] X. Zhang, C.-L. Zou, L. Jiang, and H. X. Tang, Phys. Rev. Lett. 113, 156401 (2014).

[7] Y. Tabuchi, S. Ishino, T. Ishikawa, R. Yamazaki, K. Usami, and Y. Nakamura, Phys. Rev. Lett. 113, 083603 (2014).

[8] M. Goryachev, W. G. Farr, D. L. Creedon, Y. Fan, M. Kostylev, and M. E. Tobar, Phys. Rev. Appl. 2, 054002 (2014).

[9] N. J. Lambert, J. A. Haigh, and A. J. Ferguson, J. Appl. Phys. 117, 053910 (2015).

[10] J. Bourhill, N. Kostylev, M. Goryachev, D. L. Creedon, and M. E. Tobar, Phys. Rev. B 93, 144420 (2016).

[11] M. Afzelius, I. Usmani, A. Amari, B. Lauritzen, A. Walther, C. Simon, N. Sangouard, J. Minár, H. de Riedmatten, N. Gisin, and S. Kröll, Phys. Rev. Lett. 104, 040503 (2010).

[12] N. Sangouard, C. Simon, H. de Riedmatten, and N. Gisin, Rev. Mod. Phys. 83, 33 (2011).

[13] N. Timoney, I. Usmani, P. Jobez, M. Afzelius, and N. Gisin, Phys. Rev. A 88, 022324 (2013).

[14] C.-H. Lambert, S. Mangin, B. S. D. C. S. Varaprasad, Y. K. Takahashi, M. Hehn, M. Cinchetti, G. Malinowski, K. Hono, Y. Fainman, M. Aeschlimann, and E. E. Fullerton, Science 345, 1337 (2014).

[15] P. Jobez, I. Usmani, N. Timoney, C. Laplane, N. Gisin, and M. Afzelius, New J. Phys. 16, 083005 (2014).

[16] P. Jobez, C. Laplane, N. Timoney, N. Gisin, A. Ferrier, P. Goldner, and M. Afzelius, Phys. Rev. Lett. 114, 230502 (2015).

[17] M. Gündoğan, P. M. Ledingham, K. Kutluer, M. Mazzera, and H. de Riedmatten, Phys. Rev. Lett. 114, 230501 (2015).
[18] X. Zhang, C.-L. Zou, N. Zhu, F. Marquardt, L. Jiang, and H. X. Tang, Nat. Commun. 6, 8914 (2015).

[19] D. Lachance-Quirion, Y. Tabuchi, A. Gloppe, K. Usami, and Y. Nakamura, Appl. Phys. Express 12, 070101 (2019).

[20] R. Hisatomi, A. Osada, Y. Tabuchi, T. Ishikawa, A. Noguchi, R. Yamazaki, K. Usami, and Y. Nakamura, Phys. Rev. B 93, 174427 (2016).

[21] J. Hesler, R. Prasankumar, and J. Tignon, J. Appl. Phys. 126, 110401 (2019)

[22] G. Scalari, C. Maissen, D. Turčinková, D. Hagenmüller, S. De Liberato, C. Ciuti, C. Reichl, D. Schuh, W. Wegscheider, M. Beck, and J. Faist, Science 335, 1323 (2012).

[23] X. Li, M. Bamba, Q. Zhang, S. Fallahi, G. C. Gardner, W. Gao, M. Lou, K. Yoshioka, M. J. Manfra, and J. Kono, Nat. Photonics 12, 324 (2018).

[24] G. L. Paravicini-Bagliani, F. Appugliese, E. Richter, F. Valmorra, J. Keller, M. Beck, N. Bartolo, C. Rössler, T. Ihn, K. Ensslin, C. Ciuti, G. Scalari, and J. Faist, Nat. Phys. 15, 186 (2019).

[25] P. Sivarajah, A. Steinbacher, B. Dastrup, J. Lu, M. Xiang, W. Ren, S. Kamba, S. Cao, and K. A. Nelson, J. Appl. Phys. 125, 213103 (2019).

[26] M. Białek, A. Magrez, and J.-P. Ansermet, Phys. Rev. B 101, 024405 (2020).

[27] F. Keffer and C. Kittel, Phys. Rev. 85, 329 (1952).

[28] O. Gomonay, V. Baltz, A. Brataas, and Y. Tserkovnyak, Nat. Phys. 14, 213 (2018).

[29] T. Jungwirth, J. Sinova, A. Manchon, X. Marti, J. Wunderlich, and C. Felser, Nat. Phys. 14, 200 (2018).

[30] M. B. Jungfleisch, W. Zhang, and A. Hoffmann, Phys. Lett. A 382, 865 (2018).

[31] V. Baltz, A. Manchon, M. Tsoi, T. Moriyama, T. Ono, and Y. Tserkovnyak, Rev. Mod. Phys. 90, 015005 (2018).

[32] M. Mergenthaler, J. Liu, J. J. Le Roy, N. Ares, A. L. Thompson, L. Bogani, F. Luis, S. J. Blundell, T. Lancaster, A. Ardavan, G. A. D. Briggs, P. J. Leek, and E. A. Laird, Phys. Rev. Lett. 119, 147701 (2017).

[33] Y. Xiao, X. H. Yan, Y. Zhang, V. L. Grigoryan, C. M. Hu, H. Guo, and K. Xia, Phys. Rev. B 99, 094407 (2019). 
[34] Ø. Johansen and A. Brataas, Phys. Rev. Lett. 121, 087204 (2018).

[35] T. Higuchi and M. Kuwata-Gonokami, Nat. Commun. 7, 10720 (2016).

[36] P. Němec, M. Fiebig, T. Kampfrath, and A. V. Kimel, Nat. Phys. 14, 229 (2018).

[37] J. A. Haigh, S. Langenfeld, N. J. Lambert, J. J. Baumberg, A. J. Ramsay, A. Nunnenkamp, and A. J. Ferguson, Phys. Rev. A 92, 063845 (2015).

[38] A. Osada, A. Gloppe, R. Hisatomi, A. Noguchi, R. Yamazaki, M. Nomura, Y. Nakamura, and K. Usami, Phys. Rev. Lett. 120, 133602 (2018).

[39] A. Osada, A. Gloppe, Y. Nakamura, and K. Usami, New J. Phys. 20, 103018 (2018).

[40] T. Liu, X. Zhang, H. X. Tang, and M. E. Flatté, Phys. Rev. B 94, 060405(R) (2016).

[41] E. Almpanis, Phys. Rev. B 97, 184406 (2018).

[42] P. A. Pantazopoulos, N. Stefanou, E. Almpanis, and N. Papanikolaou, Phys. Rev. B 96, 104425 (2017).

[43] P. A. Pantazopoulos, K. L. Tsakmakidis, E. Almpanis, G. P. Zouros, and N. Stefanou, New J. Phys. 21, 095001 (2019).

[44] S. Viola Kusminskiy, H. X. Tang, and F. Marquardt, Phys. Rev. A 94, 033821 (2016).

[45] J. Graf, H. Pfeifer, F. Marquardt, and S. Viola Kusminskiy, Phys. Rev. B 98, 241406(R) (2018).

[46] S. Sharma, Y. M. Blanter, and G. E. W. Bauer, Phys. Rev. Lett. 121, 087205 (2018).

[47] V. A. S. V. Bittencourt, V. Feulner, and S. V. Kusminskiy, Phys. Rev. A 100, 013810 (2019).

[48] M. Aspelmeyer, T. J. Kippenberg, and F. Marquardt, Rev. Mod. Phys. 86, 1391 (2014).

[49] R. J. Powell and W. E. Spicer, Phys. Rev. B 2, 2182 (1970).

[50] M. J. Dodge, Appl. Opt. 23, 1980 (1984).

[51] I. R. Jahn, Phys. Status Solidi B 57, 681 (1973).

[52] C. Kittel, Quantum Theory of Solids, 2nd ed. (Wiley, New York, 1963).

[53] T. Holstein and H. Primakoff, Phys. Rev. 58, 1098 (1940).

[54] See Supplemental Material at http://link.aps.org/supplemental/ 10.1103/PhysRevResearch.2.022027 for (I) details on the diagonalization of the AFM Hamiltonian in terms of the Bogoliubov modes following Refs. [34,55], (II) a derivation of the optomagnonic Hamiltonian for antiferromagnetic systems starting with the Hamiltonian from Refs. [58,59,87], (III) symmetry considerations for deriving the properties of the optomagnonic coupling, and (IV) a detailed derivation of the cavity spectra from the Heisenberg-Langevin equations [74,88-92] and the role of cavity-induced magnon-magnon interactions on dynamical quantities.

[55] A. Kamra, U. Agrawal, and W. Belzig, Phys. Rev. B 96, 020411(R) (2017).

[56] A. Kamra and W. Belzig, Phys. Rev. Lett. 119, 197201 (2017).

[57] F. L. A. Machado, P. R. T. Ribeiro, J. Holanda, R. L. RodríguezSuárez, A. Azevedo, and S. M. Rezende, Phys. Rev. B 95, 104418 (2017).

[58] M. G. Cottam, J. Phys. C: Solid State Phys. 8, 1933 (1975).

[59] M. G. Cottam and D. J. Lockwood, Light Scattering in Magnetic Solids (Wiley, New York, 1986).

[60] D. J. Lockwood and M. G. Cottam, Low Temp. Phys. 38, 549 (2012).
[61] J. Ariai, P. A. Bates, M. G. Cottam, and S. R. P. Smith, J. Phys. C: Solid State Phys. 15, 2767 (1982).

[62] L. Bi, A. R. Taussig, H.-S. Kim, L. Wang, G. F. Dionne, D. Bono, K. Persson, G. Ceder, and C. A. Ross, Phys. Rev. B 78, 104106 (2008).

[63] S. Sharma, B. Z. Rameshti, Y. M. Blanter, and G. E. W. Bauer, Phys. Rev. B 99, 214423 (2019).

[64] N. Brahms and D. M. Stamper-Kurn, Phys. Rev. A 82, 041804(R) (2010).

[65] J. Kohler, N. Spethmann, S. Schreppler, and D. M. StamperKurn, Phys. Rev. Lett. 118, 063604 (2017).

[66] M. Landini, N. Dogra, K. Kroeger, L. Hruby, T. Donner, and T. Esslinger, Phys. Rev. Lett. 120, 223602 (2018).

[67] R. M. Kroeze, Y. Guo, V. D. Vaidya, J. Keeling, and B. L. Lev, Phys. Rev. Lett. 121, 163601 (2018).

[68] F. Mivehvar, H. Ritsch, and F. Piazza, Phys. Rev. Lett. 122, 113603 (2019).

[69] J. Barak, V. Jaccarino, and S. Rezende, J. Magn. Magn. Mater. 9, 323 (1978).

[70] R. C. Ohlmann and M. Tinkham, Phys. Rev. 123, 425 (1961).

[71] T. Satoh, S.-J. Cho, R. Iida, T. Shimura, K. Kuroda, H. Ueda, Y. Ueda, B. A. Ivanov, F. Nori, and M. Fiebig, Phys. Rev. Lett. 105, 077402 (2010).

[72] T. Kampfrath, A. Sell, G. Klatt, A. Pashkin, S. Mährlein, T. Dekorsy, M. Wolf, M. Fiebig, A. Leitenstorfer, and R. Huber, Nat. Photonics 5, 31 (2011).

[73] S. Zhou, Y. Gao, and S. Fu, Eur. Phys. J. B 91, 41 (2018).

[74] S. Weis, R. Rivière, S. Deléglise, E. Gavartin, O. Arcizet, A. Schliesser, and T. J. Kippenberg, Science 330, 1520 (2010).

[75] H. Xiong and Y. Wu, Appl. Phys. Rev. 5, 031305 (2018).

[76] C. Genes, D. Vitali, and P. Tombesi, New J. Phys. 10, 095009 (2008).

[77] C. F. Ockeloen-Korppi, M. F. Gely, E. Damskägg, M. Jenkins, G. A. Steele, and M. A. Sillanpää, Phys. Rev. A 99, 023826 (2019).

[78] C. Sommer and C. Genes, Phys. Rev. Lett. 123, 203605 (2019).

[79] S. A. McGee, D. Meiser, C. A. Regal, K. W. Lehnert, and M. J. Holland, Phys. Rev. A 87, 053818 (2013).

[80] M. D. Lukin, S. F. Yelin, and M. Fleischhauer, Phys. Rev. Lett. 84, 4232 (2000).

[81] M. Fleischhauer and M. D. Lukin, Phys. Rev. Lett. 84, 5094 (2000).

[82] M. Fleischhauer and M. D. Lukin, Phys. Rev. A 65, 022314 (2002).

[83] A. V. Gorshkov, A. André, M. D. Lukin, and A. S. Sørensen, Phys. Rev. A 76, 033804 (2007).

[84] L. Ma, O. Slattery, and X. Tang, J. Opt. 19, 043001 (2017).

[85] H. Kondoh and T. Takeda, J. Phys. Soc. Jpn. 19, 2041 (1964).

[86] J. Baruchel, M. Schlenker, K. Kurosawa, and S. Saito, Philos. Mag. B 43, 853 (1981).

[87] L. D. Landau and E. M. Lifshitz, Electrodynamics of Continuous Media, 2nd ed. (Pergamon, Oxford, UK, 1984).

[88] C. W. Gardiner and P. Zoller, Quantum Noise: A Handbook of Markovian and Non-Markovian Quantum Stochastic Methods with Applications to Quantum Optics, 2nd ed. (Springer, Berlin, 2000). 
[89] S. M. Spillane, T. J. Kippenberg, O. J. Painter, and K. J. Vahala, Phys. Rev. Lett. 91, 043902 (2003).

[90] M. Cai, O. Painter, and K. J. Vahala, Phys. Rev. Lett. 85, 74 (2000).
[91] T. P. Purdy, P.-L. Yu, R. W. Peterson, N. S. Kampel, and C. A. Regal, Phys. Rev. X 3, 031012 (2013).

[92] A. H. Safavi-Naeini, T. P. M. Alegre, J. Chan, M. Eichenfield, M. Winger, Q. Lin, J. T. Hill, D. E. Chang, and O. Painter, Nature (London) 472, 69 (2011). 